INTERNATIONAL DESIGN CONFERENCE - DESIGN 2018

https://doi.org/10.21278/idc.2018.0506

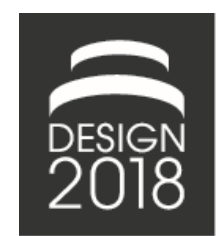

\title{
DESIGN FOR HEALTH: TOWARDS COLLABORATIVE CARE
}

\author{
J. F. Valentin-Hjorth, F. Patou, N. Syhler, H. Dominguez and A. Maier
}

\begin{abstract}
The design of novel healthcare delivery models better suited to address the burden of chronic diseases requires a thorough understanding of the foundational concepts of patient and healthcare provider collaboration. Reviewing the literature, we propose a taxonomy towards collaborative care: a generic term characterising healthcare delivery models that focus on the importance of patient-provider interactions, support safe patient participation in their own care, and redefine the balance of decisionpower and accountability between patient and provider in health and care management.
\end{abstract}

Keywords: healthcare design, digital design, participation, patient engagement, systems engineering (SE)

\section{Introduction}

Our national healthcare systems face unprecedented challenges. World population ageing and associated growth of incidences and prevalence of chronic diseases weigh heavy on healthcare budgets. Increasing costs of technology adoption stretch the economy of health further and care personnel scarcity threatens the foundations on which most modern national healthcare systems are built: equity of access to care and high value delivery (Patou and Maier, 2017).

The proliferation of Information and Communication Technology (ICT) in the 1980s and the accelerating pace of innovation in this domain have nourished continuous hope in our ability to cope with the aforementioned challenges for health services and care provision. Since the early emergence of Hospital Information Systems (HIS) and following more recent developments in mobile Health (mHealth), wearable technology, and Artificial Intelligence, ICT innovation has allowed the conceptualisation and implementation of new healthcare delivery models meant to increase both care efficacy and cost- and resource efficiency (Sclafani et al., 2013; Weinhold and Gastaldi, 2015).

A significant number of other ICT solutions and product-services that also were and are meant to provide patients with the ability to become prominent contributors to their own care management have been conceptualised, developed and tested (McColl et al., 2014; Pirolli et al., 2017; Thorpe et al., 2017). Yet, studies investigating how these solutions perform have unveiled persistent shortcomings (Barello et al., 2016; Bloss et al., 2016). Central in the discussion of why many such studies failed to deliver and measure-up to their expectations, is evidence of the difficulty in maintaining patient engagement and continuous effort over the long periods of time (Dennison et al., 2013; Paz Castro et al., 2017). Recently, for instance, Kim et al. (2016) documented in their review that almost $50 \%$ of trial participants in a web-based mobile health study only used the tool they were provided with once. 
Using a smartphone-based solution coupled to a number of health self-trackers, Bloss et al. (2016), conducted a randomised controlled trial over a six months period, investigating the short-term effects of mHealth self-monitoring on patients suffering from a variety of chronic diseases. Results from the study disappointingly showed no significant difference between intervention and control group in terms of economic value or health outcome.

These setbacks have raised a number of questions to the shortcomings of healthcare models relying on patient self-care. It has therefore been suggested that technology alone only serves as a tool to enable patients to get better care, but cannot drive motivation towards a drastic shift in accountability and participation. Angel and Frederiksen (2015), for example, have argued that the success of newly proposed healthcare models is hindered by poor patient-provider collaboration and that better collaboration between the two main actors of the care process is needed. With the help of well-designed ICT solutions, better care models ought to help transform patients from being "passive recipients" to "active decision-makers" (Pravettoni and Gorini, 2011). The proposition is that as patients become more prominent, accountable, and informed contributors of their own health management, various benefits will ensue: self-efficacy, proactivity, a better life-style preventing the development of chronic diseases such as diabetes or hypertension and their comorbidities, lower demands on the availability of care personnel, better treatment adherence, higher patient satisfaction for care procedures performed at home, financial savings of fewer hospitalisations, and more:

An emergence of such collaborative healthcare models would come with its set of challenges as it implies a profound change in the dynamic that has traditionally characterised interactions between patients and healthcare professionals, challenging what some have called the patriarchal model of care delivery (Gruman et al., 2010; Twine Health, 2016) and encourage instead a shift towards patient empowerment, engagement, self-management, and generally higher health literacy. Patient-centred care was suggested almost two decades ago with these objectives in mind, putting patient needs and desires at the centre of the care delivery process. Patient-centred care also advocates that patients become active participants, rather than passive receivers of care (Mead and Bower, 2000; Stewart et al., 2003; Constand et al., 2014).

Where patient-centred care mainly targets the processes and organisational changes required for active patient participation, instead, what we call Collaborative Care focuses on direct patient-provider interactions and exchanges. We thus see Collaborative Care as necessary complement to current patientcentred care approaches in view of rebalancing patient-provider relations, including the move towards redefining expected health-related knowledge levels on both sides, towards more patient involvement in planning and therapy, and towards the realignment of the amount of ownership and accountability between both parties.

Opportunities for designers looking to support the conceptualisation of novel and improved models of collaborative healthcare are thus numerous. At this point in time, collaboration is merely understood as an umbrella term and the implementation of collaborative care models is made difficult by the absence of a clear taxonomy of the concepts the term spans across. Health literature is replete in regards to studies on increased patient-provider collaboration, yet definitions for these terms are rarely aligned and are often applied interchangeably. Understanding of the concepts also keeps evolving as new technologies and other healthcare delivery models are proposed.

To enhance patient-provider relations and motivation towards what we term Collaborative Care, this paper investigates the concepts and interrelations of enablement, empowerment, engagement, involvement, and participation by reviewing health sciences literature regarding challenges to patientprovider collaboration and the characterisation of the abovementioned concepts and their potential interrelations (see also Figure 1). The concepts included in the taxonomy are the ones we envisage as the starting point for designing future collaborative care models.

The remainder of the paper is structured as follows. Section 2 proposes a taxonomy towards Collaborative Care, discussing the core concepts enablement, empowerment, engagement, involvement, participation and their interrelations and emphasises the role of shared decision making towards achieving Collaborative Care. By collaborative care we understand direct patient-provider interactions and exchanges that occur on multiple occasions during (chronic) care management. Section 3 crystallises what is needed to achieve collaborative care. In Section 4, avenues for future research for 
design for health towards collaborative care and implications for healthcare practitioners are discussed, followed by conclusions in Section 5.

\section{A taxonomy of collaborative care}

\subsection{Understanding collaborative care}

As a first step towards forming a taxonomy of collaborative care, we start by looking into what collaboration may encompass in general and what has been mentioned in the healthcare patient-provider context in particular. The Merriam-Webster (2017) dictionary defines collaboration as "to work jointly with others or together especially in an intellectual endeavour".

In the healthcare context and according to Henneman et al. (1995), for collaboration to occur, "two or more individuals must be involved in a joint venture, typically one of an intellectual nature [...] in which participants willingly participate in planning and decision making". This, so the authors further highlight, includes sharing power and responsibility on the basis of knowledge and expertise towards a common goal as well as being members of a team without roles, titles or hierarchy. Cahill (1996) also argues that this way of balancing power creates a greater sense of trust, respect, and willingness, from where better relationships emerge. These characteristics are important notions of the patient-provider relationship, which we will discuss further on in the paper.

In their review of a patient-provider collaboration, Carnwell and Buchanan (2004) summarise the attributes of collaboration as the following: Intellectual and co-operative endeavour, knowledge and expertise more important than role or title, joint venture, team working, participation in planning and decision making, a non-hierarchical relationship, sharing of expertise, willingness to work together towards an agreed purpose, trust and respect in collaborators, highly connected network, and low expectation of reciprocation. Carnwell and Buchanan (2004) point in particular to a related concept to collaboration, namely partnership. In their eyes, a patient-provider partnership means "shared commitment, where all partners have a right and an obligation to participate and will be affected equally by the benefits and disadvantages arising from the partnership“. In that sense, patient-provider relationships are not partnerships, but are characterised by a number of attributes Carnwell and Buchanan (2004) associate with collaboration.

The term collaborative care is scarcely applied in current research and where it is, it is used in multiple ways: In one study, "Collaborative Care" is described as 'patient interventions' that are providerdriven, and include education, legal and financial advice and 'guidance tools' as a minimum (Callahan et al., 2006). To examine quality indicators in palliative and end-of-life care, Pfaff and Markaki (2017) seek to define a framework they call "Compassionate Collaborative Care", where collaboration takes the form of a synergistic group effort aimed at better determining needs and value for patients, but also families and healthcare providers. In a third study, the formulation "Collaborative Care" is not defined as a healthcare delivery model but is used as a way to describe the addition of care management nurses to "Collaborative Care Teams" (Katon et al., 2010). In yet another study, Belyeu et al. (2017) found that $45 \%$ of participants lacked engagement in participating in the "Diabetes Collaborative Care Team Program" the authors of the study had set up. What the studies have in common is the conclusion that when patients do not engage in assigned collaborations, patients are less accountable and adherent to treatment models. And this is an issue as sustained health improvements can then not be documented.

We thus argue in this paper that collaborative care models need to (1) rebalance power, knowledge and legitimacy in a patient-provider relationship and (2) also encourage and support engagement and participation of patients, so that accountability is increased and healthcare outcomes are bettered. Collaborative Care thus focuses on the direct patient-provider collaboration and interactions that occur on multiple occasions during chronic care management, rather than process components on which patient-centred care focuses (Constand et al., 2014). 


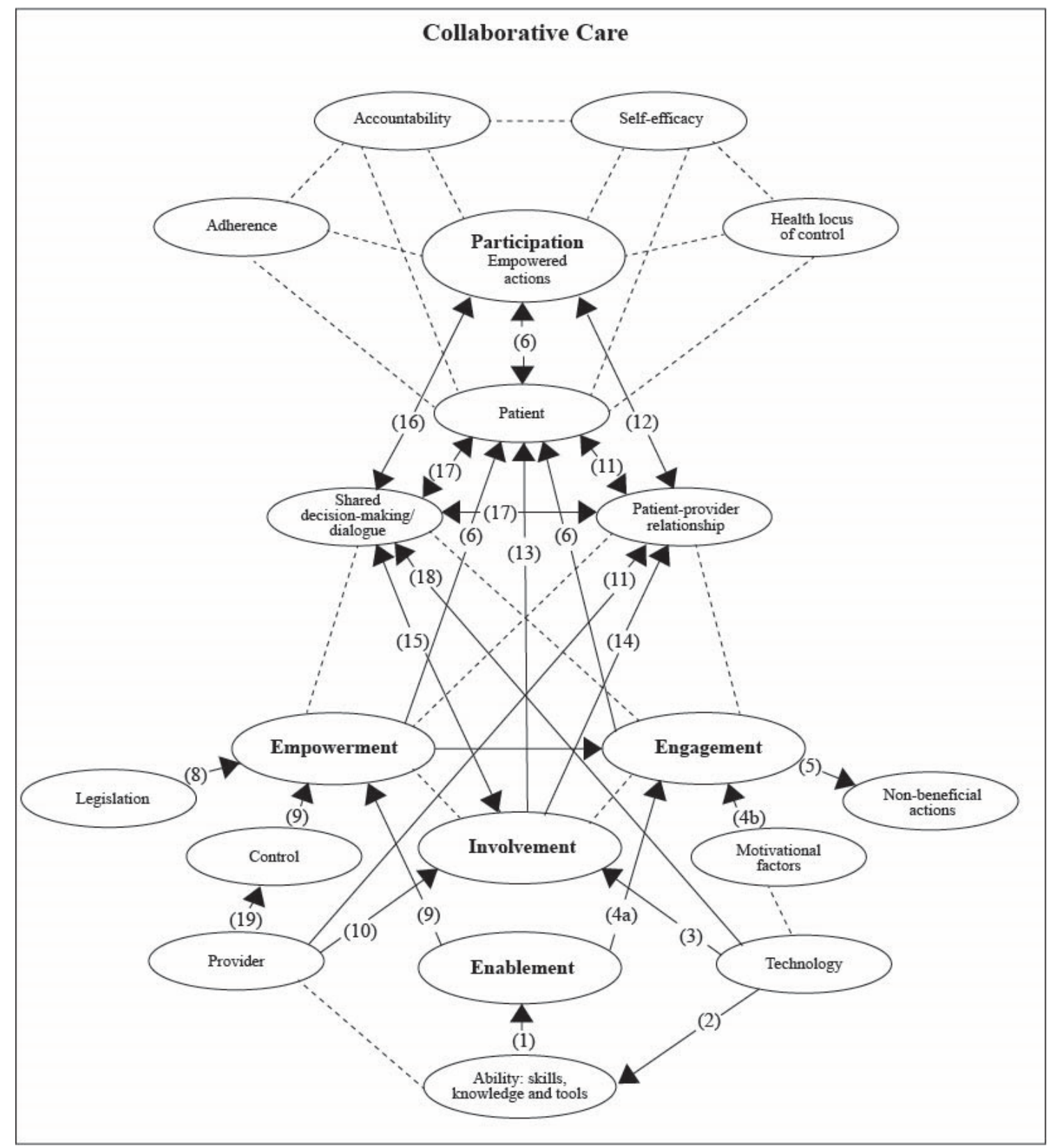

Figure 1. A taxonomy of collaborative care

\subsection{Composing collaborative care}

Researchers in the health sciences have investigated a number of concepts associated with patientprovider collaboration. In particular, there appears to be a significant difference between being able to participate in healthcare treatment and being engaged to be actively doing so. The most prominent concepts we identified from the literature are: enablement, engagement, empowerment, involvement, and participation. Using these terms as keywords in our literature search, we examined the concepts and affiliated connotations for these terms. Based on what was stated in the articles we studied, we then identified and listed associations between concepts, as well as discrepancies or contradictions. The concepts, their potential relations as per literature, and associated terms such as, for example, shared decision making, are reviewed and discussed in the following section. Based on literature, Figure 1 proposes a taxonomy towards collaborative care and visually illustrates the concepts and their 
connections in the healthcare context. The mapping of concepts as depicted in Figure 1 should be looked upon as the groundwork for further investigations, creating the base and opportunity for translation into tools for implementing Collaborative Care in practice.

The taxonomy is described as built-up in what follows. We discuss concepts presented in literature as we believe they were intended by the authors in single quotation marks ('term'), while we refer to our own definition for these concepts using italics (term). Connections are referred to with numbers in parentheses (1 through to 19) and visualised as annotated arrows in Figure 1. Dotted lines indicate relations that are not discussed in detail in this paper.

Starting with (1), in previously described studies (e.g. McColl et al., 2014; Pirolli et al., 2017) 'ability' was defined as the provision of tools or interventions to monitor, diagnose and treat patients. However, 'ability' not only require tools, it also necessitates the competencies to use them and the understanding of the necessity to do so. In their review of 'empowerment' and neighbouring terms, Fumagalli et al. (2015) describe the acquisition of 'ability' as the development of "skills and knowledge through enablement", what we agree with: enablement evokes the acquisition of skills and knowledge related to one's health and conditions (1). Digital technology can help to acquire and later apply these skills and knowledge when appropriate (2,3, and 18). Thus, in order to enable patients, health information needs to be translated into actionable knowledge for patients.

Recognising the importance of patients' skills and knowledge in regards to their own disease is "the fundamental principle behind patient engagement" according to Duffett (2017, p. 114). By this, the authors describe patients' insights on own well-being, bodies and lives, as well as the acknowledgement of their own ability to make an impact as a drivers towards 'engagement'. The degree to which people believe they have an influence on their own health outcomes can be described as 'health locus of control', and patients who have a high level of confidence in their own abilities are more likely to be willing to take an active part in their own treatment. This is consistent with some of the findings from Bloss, et al.'s (2016) randomised control study: Even though no significant difference between the intervention and control groups was demonstrated, some individuals engaged slightly more in managing their own health, presumably because they were able to understand that their actions impacted their condition (4a). However, this only applied for a few participants and was thus not sufficient to carry overall participation. Thus, an increased health locus of control is an important but not necessarily sufficient driver for 'engaging' patients; motivation to take action is also needed, and motivating patient engagement must be nurtured continuously in order to maintain levels of patient participation in long-term self-care. Fumagalli et al. (2015) describe 'engagement' as a mean towards the acquisition of motivation - a positive attitude change as well as self-awareness in regards to health locus of control. In addition to an increased self -awareness, a positive attitude towards selfmanagement is thus also a part of 'engagement' as are 'motivational factors'. We expand this description and argue that engagement is the notion of actively taking part in one's own health treatment through motivation (4b). However, patients can be motivated to engage in their own treatment without either having power to do so or the knowledge to understand the potential consequences of their actions. When patients are active but non-informed the outcome of self-care might be poor (5). Thus, patients not only need to be enabled with tools, skills and knowledge, or engaged through motivational factors to have the best base of self-care, they also need to have the power to do so.

According to Fumagalli et al.'s (2015) terminology, 'empowered' patients are simultaneously 'engaged' and 'enabled'. They are "'willing and able' to play an active role in their care", thus providing an ideal ground for collaboration. The authors thus describe 'empowerment' as the combination of the acquisition of motivation and enablement, i.e. the acquisition of ability - skills, knowledge and tools. In contrast to Fumagalli et al. (2015), we argue that engagement is not an attribute of empowerment. Indeed, possessing any kind of 'power' does not necessarily mean using it. We argue instead that, one needs to be motivated in using one's power. Engagement added to empowerment should therefore lead to patients taking part of what we will later define as empowered action or participation (6). Furthermore, we contend that the notion of empowerment in the healthcare context does not only rely on the availability of tools, but also evokes development of skills, transformation of information to knowledge (7), legitimacy (8), and control over the course actions related to one's health (9). 
Consequently, patient enablement does not suffice in driving collaborative care. Likewise, empowerment is needed but does not necessarily come with the motivational charge needed to engage in participation. It follows from this conceptualisation that in order to increase accountability of patients, collaborative care models must continuously promote patient empowerment, while generating motivation as a driver of engagement and self-efficacy. Persistence of effort is key, since chronic healthcare can be looked upon as a continued process, where iterations makes patients become more involved and more accountable for each step.

Engagement and enablement are tightly connected to patient involvement and participation. We concur that involvement is to be regarded as provider-driven, applicable when motivation is not already present in patients (10). Providers determine, in consideration of their patients' level of empowerment and the extent to which they want to involve them. 'Participation' is driven by both patient and provider and healthcare goals are co-determined (11) (Cahill, 1996; Thompson, 2007). Possibilities of participation are created when balance in patient-provider relationship is pursued and alliances of "trust, respect and willingness" are increasingly developed (12) (Carnwell and Buchanan, 2004). Thus, involvement - of patients (13) by providers - is a precursor to participation and can be applied as the initial gateway to establishing a successful patient-provider relationship (14).

Thompson (2007) discusses the level (amount) of collaboration desired by patients - their willingness to collaborate - in regards to 'involvement' and 'participation'. For the author, 'participation' encompasses 'shared decision-making' (SDM) and 'dialogue', with SDM in medicine representing a higher level of collaboration than dialogue. In this sense, dialogue can be seen as a low-level collaboration intervention, suited for early involvement of the patient in the collaborative process (15), whereas SDM can be applied towards participation (16). Literature on the subject implies that shared decision-making leads to increased adherence and encouragement to self-sufficiency by integration of each patient's individual needs (Friesen-Storms et al., 2015). However, others have implied that SDM faces obstacles either inherent to the organization of most healthcare system models or to the given intervention, or extrinsic such as an insufficient amount of time in consultation, or power imbalances between patients and healthcare professionals. These barriers put the creation of an effective collaborative space at risk, especially when trust and respect are missing (Forbat et al., 2009; Joseph-Williams et al., 2014). Angel and Frederiksen's (2015) review on 'Challenges in Achieving Patient Participation' also describes the nature of patient-provider relationship as a challenge. They contend that in order to facilitate patient participation, it is necessary to create a mutual relationship of trust and acknowledgement between patient and provider, where knowledge and understanding is created from a two-way sharing of information, similar to SDM (17).

Moreover, healthcare professionals and caregivers' attitude towards patient participation is crucial in developing these relations, and the shaping of this attitude is just as important as it is to promote engagement and empowerment on the patient side. Furthermore, time limitations and patient's age, disease severity and cognitive skills influence the development of successful relationships: When time is limited, there is a risk of caregivers or healthcare professionals not putting energy into two-way conversations or relationship development. Old age, severe illness, poor health, and varying health literacy level also present poor conditions for patient participation, since patients presenting any of these factors might not be physically or cognitively able to participate. Patient participation should be encouraged as a function of physical and mental capabilities. However, patients may unjustifiably suffer from negative self-perception of their ability to participate. Hibbard and Mahoney (2010) describe negative 'self-conception' as the reason to low activation in patients, where self-conception refers to patients' perception on self-sufficiency. By settling for smaller steps in treatment milestones/goals, interventions that inspire positive emotions are created, so that it is more likely for a patient to reach success, resulting in a positive spiral of patient self-perception. We further lean from literature that shared decision-making (SDM) interventions should facilitate patient participation. This requires not only patients' willingness and physical/mental ability to collaborate, but also a mind-set and attitude on the provider side favourable to balanced collaboration and sharing of control (19). It also demands wellthought through framing and sufficient time to establish trustful relationships. Additionally, we defined patient involvement as the deliberate inclusion by providers of patients in the patient's own health management. For this, insights on patients' wishes and needs should be examined, e.g. through dialogue, 
in order to set initial treatment milestones that can increase the locus of health and motivation when reached.

In summary, through the review we have come to understand the core concepts of a taxonomy towards collaborative care in the following way:

- Enablement: the process of acquisition of health-related abilities, whether in the form of skills or knowledge, potentially through the use of tools such as ICT solutions.

- Engagement: the degree to which a patient is willing to participate in the care delivery process.

- Empowerment: the process encompassing enablement, as well as that of gaining both control over the patient's own health decision-making and legitimacy. Empowerment, therefore, involves becoming able to participate in one's own care but also becoming juristically and morally allowed to do so.

- Involvement: the degree of participation that providers actively attempt to obtain from patients. The degree of involvement is thus in part determined by how legitimate and appropriate a provider believes it is to ask a patient to participate in the care delivery process.

- Participation: The tangible actions and behaviours exhibited by patients with the aim of benefiting their own health. As such participation can be beneficial if it is driven by both the patient and the provider, i.e. the result of engagement and involvement, or undertaken by an empowered and engaged patient. Participation can however also have adverse effects if undertaken by patients who lack skills and knowledge without being overseen by providers.

In addition to the core concepts like enablement, empowerment, engagement, involvement, and participation, a number of connected concepts have emerged during the examination of health sciences literature towards new healthcare delivery models, with shared decision-making, patientprovider relationship and actions as examples. To complete the description of Figure 1, some connections (dotted) have not been directly defined in this section, but have been discussed earlier or are inferred connections. It has earlier been argued that better patient-provider collaboration creates better health outcomes such as adherence, accountability, self-efficacy and health locus of control, which necessarily also need to connect to the patient. Additionally, it is reasonable to believe that empowerment and engagement have two-way connections to shared decision-making SDM)/dialogue and patient-provider relationships, seeing that balanced relationships might contribute to engagement and empowerment and vice versa. Also, engagement and empowerment can lead to SDM/dialogue and vice versa. Finally, it can be argued that motivational factors can be incorporated in the technology of ICT tools, as well as providers may have the power to equip patients with skills, knowledge, and tools.

Finally, to transform patients into "active decision-makers" in their own health treatment, it is also necessary to connect action to the concepts. First of all, we can argue that patient empowerment without the support of engagement may lead to little or no action at all (empowerment minus engagement $=>$ little/no action). Second of all, it is reasonable to argue that patients who are engaged, but lack power in terms of ability, control, or legitimacy to perform actively, cannot take any action on their own (engagement minus power $=$ no action). However engaged patients may be, they may also need to be authorised and in control to perform actively, but may still lack the ability to act towards positive outcomes from a poor base of skills and knowledge. This may lead to actions that are not beneficial to patients' treatment (engagement + control + legislation minus ability $=$ non-beneficial actions) (5). Accordingly, activity does not have an inherent value in driving collaborative care, it needs to be performed from a base of knowledge and skills as a part of empowerment in order to better treatment results (engagement + power $=$ beneficial actions). Empowered actions is thus synonymous to participation, where patients take part in shared decision-making and thus becomes "active decisionmakers".

\section{Satisfying collaborative care objectives}

Reviewing and linking concepts discussed in the health sciences in relation to patient-provider collaboration helps depicting more sharply what we believe should be the core constituents of any collaborative healthcare model. 
In order to encourage a shift towards patient empowerment and self-management, it seems that implementation of a strategy is needed for shifting the balance of power (the possession of skills, knowledge but also control and legitimacy to act) and for continuously motivating patients to engage in their own treatment. Empowerment and engagement are thus connected to patient-provider relationships: Patients that feel heard and respected are more likely to gain trust in healthcare providers, which may engage patients in co-determining treatment goals through shared decision-making. A continuity of engagement and empowerment is important to sustain and develop participation, since skills can be expanded, understanding can be deepened, and control reinforced and motivation sustained, specifically when dealing with long-term chronic diseases.

Current treatment models focus on the enablement of patients by providing tools, skills and knowledge to patients (Dennison et al., 2013; Barello et al., 2016; Bloss et al., 2016; Kim et al., 2016; Paz Castro et al., 2017). However, as long as healthcare providers hesitate to share control, patients cannot be empowered. Engagement is driven by providers supporting the sustained involvement or participation of patients, which current treatment models lack to focus on (Figure 2).

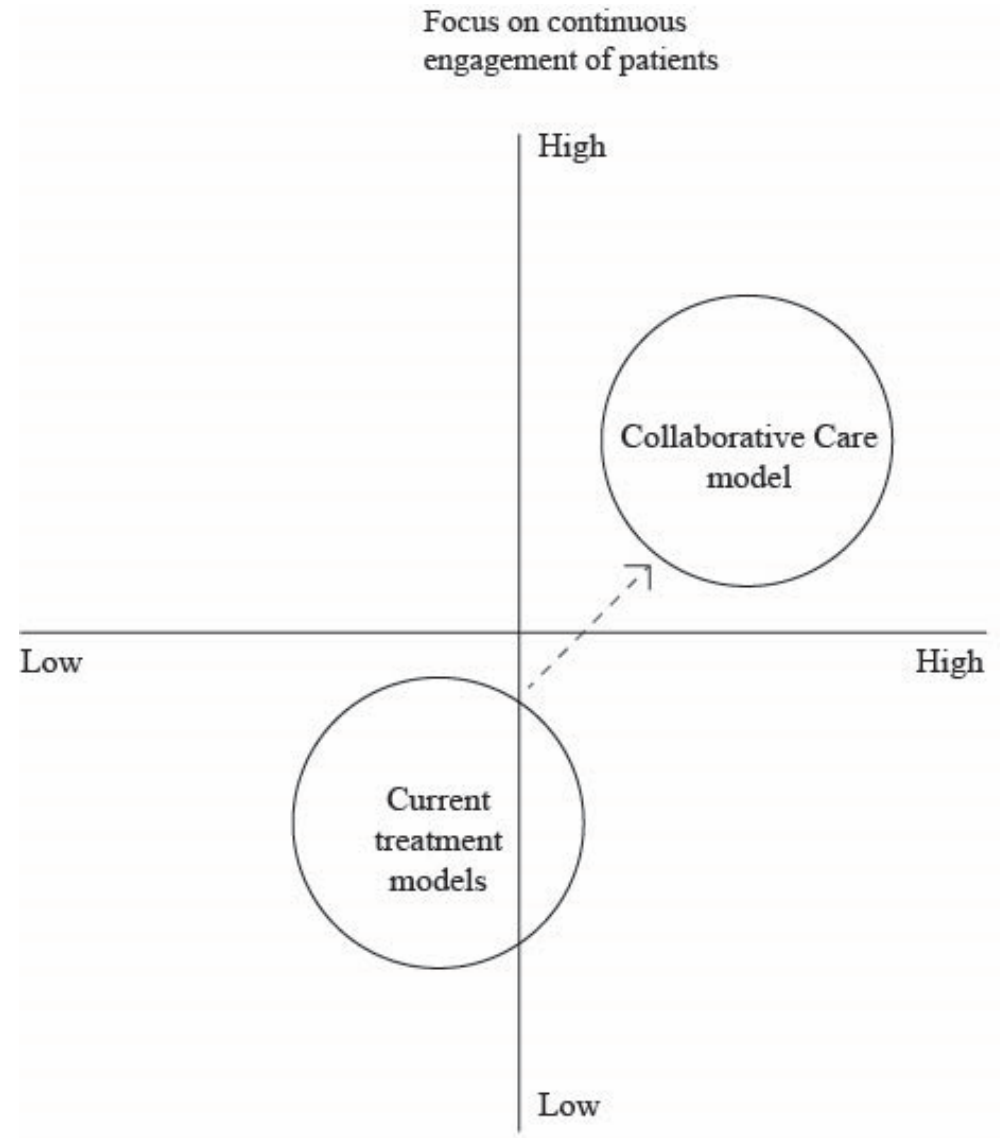

Focus on patient-provider balance (empowerment of patient)

Figure 2. Moving towards a collaborative care model with focus on a high patientprovider balance and continuous engagement of patients

This is exactly what Collaborative Care as proposed in this paper aims to achieve: Driven by the continuous support of patient engagement and a patient-provider power balance, collaborative care helps patients become more empowered and engaged to play an active role in their health and care management through their own participation. 


\section{Discussion and future research}

Collaborative care aims to resolve the issues that ICT-based solutions alone cannot address, and a taxonomy of the various concepts associated with collaborative care, such as the ones we highlight in this article, namely enablement, engagement, empowerment, involvement, and participation, will be useful in designing collaborative care frameworks. Indeed, our taxonomy reveals levers to direct the development of more concrete collaborative care models. This gives ample leeway to designers eager to contribute to the design for health, to design of behaviour change in healthcare.

\subsection{Implications for designing for collaborative care}

In order to design for patient engagement and to encourage participation, motivation is needed. This can be driven by both providers and technology, yet needs continuous support for sustained behaviour change. Thus, further investigation into design for behaviour change in healthcare with the aim to heighten motivation in self-care and -management provide a fruitful ground for further research.

Moreover, patient empowerment is a complex concept that includes the process of provision of tools, skills, knowledge and control as well as legitimacy to patients. This means that technology needs to be developed or exploited in consideration of all these elements; it means that medical information needs to be translated into skills and knowledge that is comprehensible for patients, and that the need for incentivising providers in sharing power needs to be supported and encouraged. Literature on the design and development of treatment models suggests the potential of situated-learning, where patients participate in treatment from home or other places outside of care facilities, is a lever where technology can also contribute positively (Weinhold and Gastaldi, 2015; Twine Health, 2016). This further encourages studies on empowerment and its mechanisms in regards to healthcare design promoting selfmanagement and may help patients to become more prominent contributors to their own care management.

Another avenue for further research is health interventions to promote participation. They vary and can be both simple and complex. It has been suggested that dialogue can be applied during the initial involvement of patients, whereas shared decision-making (SDM) is a tool towards participation. However, participation is not solely driven by interventions but is also dependent on patient-provider relationships based on communication and the providers' willingness to induce these changes to treatment models that support the provision of a skillset to their patients and to help motivation and general empowerment of the patients. Additionally, attention must be paid to the interventions needed to define user needs, values and wishes for collaboration, as not all patients will have the cognitive, mental or physical capability to indulge in beneficial dialogue and shared decision-making. Thus, needs elicitation and concurrent interventions need to be properly designed, also to extract information on "the resources necessary to enable [patients] to participate in the decision-making process with their physicians" (Barello et al., 2016; Thorpe et al., 2016).

\subsection{Implications for healthcare practitioners}

To move towards collaborative care, healthcare providers need to accept a shift in power balance and control of treatment goals and models. This calls for a change in attitude and standard views on what patient-provider collaborations should be like. A shift in healthcare treatment models might also have implications on legislation with policy-makers potentially acknowledging the positive impact of increased empowerment and increased self-management. Accordingly, when impacting healthcare providers, patients, health-ICT, healthcare design towards a collaborative care model calls for transformation and thus for system-level design (Jean et al., 2012; Borgiel et al., 2015; Clarkson et al., 2017; Patou and Maier, 2017).

\section{Conclusions}

This paper proposed a taxonomy towards collaborative care, highlighting the concepts of enablement, engagement, empowerment, involvement, and participation, associated terms, and their connections as discussed in the health sciences literature. We argue that collaborative care is needed to meet the 
demands healthcare systems are facing. Population ageing calls for better solutions for chronic disease management. However, evidence suggests that technological development is not a sufficient driver towards increased patient participation. Participation in healthcare and self-management of a patient's own care requires both empowerment (ability, control and legitimacy) and engagement.

Although literature provides multiple examples of studies of patient engagement, participation, and shared decision making, it also reveals the lack of strategies for designing corresponding treatment models. By reviewing commonalities and differences of core concepts, we proposed a taxonomy of collaborative care, suggesting links between what we believe should be core constituents of any healthcare model aimed at driving patient participation. Such a model should support the redefinition of the patient-provider relationship towards one that better balances patient-provider power and sustains patient engagement. As such, hopefully leading to higher levels of self-management, patient accountability, leading to better treatment outcomes, lower care costs, and less pressure on our scarce healthcare resources.

\section{Acknowledgements}

This work is a collaboration between DTU, the University of Copenhagen, and ATAH Aps and part-funded by the Copenhagen Center for Health Technology (Cachet.dk).

\section{References}

Angel, S. and Frederiksen, K.N. (2015), "Challenges in achieving patient participation: A review of how patient participation is addressed in empirical studies”, International Journal of Nursing Studies, Vol. 52 No. 9, pp. 1525-1538. https://doi.org/10.1016/j.ijnurstu.2015.04.008

Barello, S., Triberti, S., Graffigna, G., Libreri, C., Serino, S. et al. (2016), "eHealth for patient engagement: A Systematic Review", Frontiers in Psychology, Vol. 6 No. JAN. https://doi.org/10.3389/fpsyg.2015.02013

Belyeu, B., Chwastiak, L., Russo, J., Kiefer, M., Mertens, K. et al. (2017), "Barriers to Engagement in Collaborative Care Treatment of Uncontrolled Diabetes in a Safety-Net Clinic", The Diabetes Educator, Vol. 43 No. 6, pp. 621-630. https://doi.org/10.1177/0145721717739813

Bloss, C.S., Wineinger, N.E., Peters, M., Boeldt, D.L., Ariniello, L. et al. (2016), “A prospective randomized trial examining health care utilization in individuals using multiple smartphone-enabled biosensors", PeerJ, Vol. 4, pp. e1554. https://doi.org/10.7717/peerj.1554

Borgiel, K., Christophe, M. and Minel, S. (2015), "a Multi-Level Activity Analysis for Home Healthcare Ict Tool Redesign", 20th International Conference on Engineering Design (ICED 15), pp. 1-10.

Cahill, J. (1996), "Patient participation: a concept analysis", Journal of advanced nursing, Vol. 24 No. 3, pp. 561571. https://doi.org/10.1046/j.1365-2648.1996.22517.x

Callahan, C.M., Boustani, M.A., Unverzagt, F.W., Austrom, M.G., Damush, T.M. et al. (2006), “Effectiveness of Collaborative Care for Older Adults With Alzheimer Disease in Primary Care", Jama, Vol. 295 No. 18, pp. 2148-2157. https://doi.org/10.1001/jama.295.18.2148

Carnwell, R. and Buchanan, J. (2004), Effective practice in health and social care: a partnership approach, Open University Press.

Clarkson, J. et al. (2017), Engineering better care a systems approach to health and care design and continuous improvement, Royal Academy of Engineering.

Constand, M.K., MacDermid, J.C., Dal Bello-Haas, V. and Law, M. (2014), "Scoping review of patient-centered care approaches in healthcare", BMC Health Services Research, Vol. 14 No. 1, pp. 1-9. https://doi.org/10.1186/1472-6963-14-271

Dennison, L., Morrison, L., Conway, G. and Yardley, L. (2013), “Opportunities and Challenges for Smartphone Applications in Supporting Health Behavior change: Qualitative Study”, Journal of Medical Internet Research, Vol. 15 No. 4, pp. e86. https://doi.org/10.2196/jmir.2583

Duffett, L. (2017), "Patient engagement: What partnering with patient in research is all about", Thrombosis Research, Vol. 150, pp. 113-120. https://doi.org/10.1016/j.thromres.2016.10.029

Forbat, L., Cayless, S., Knighting, K., Cornwell, J. and Kearney, N. (2009), "Engaging patients in health care: An empirical study of the role of engagement on attitudes and action", Patient Education and Counseling, Vol. 74 No. 1, pp. 84-90. https://doi.org/10.1016/j.pec.2008.07.055

Friesen-Storms, J.H.H.M., Bours, G.J.J.W., van der Weijden, T. and Beurskens, A.J.H.M. (2015), "Shared decision making in chronic care in the context of evidence based practice in nursing", International Journal of Nursing Studies, Vol. 52 No. 1, pp. 393-402. https://doi.org/10.1016/j.ijnurstu.2014.06.012 
Fumagalli, L.P., Radaelli, G., Lettieri, E., Bertele', P. and Masella, C. (2015), "Patient Empowerment and its neighbours: Clarifying the boundaries and their mutual relationships", Health Policy, Vol. 119 No. 3, pp. 384 394. https://doi.org/10.1016/j.healthpol.2014.10.017

Gruman, J., Rovner, M.H., French, M.E., Jeffress, D., Sofaer, S. et al. (2010), "From patient education to patient engagement: Implications for the field of patient education", Patient Education and Counseling, Vol. 78 No. 3, pp. 350-356. https://doi.org/10.1016/j.pec.2010.02.002

Henneman, E.A., Lee, J.L. and Cohen, J.I. (1995), "Collaboration: a concept analysis", Journal of Advanced Nursing, Vol. 21 No. 1, pp. 103-109. https://doi.org/10.1046/j.1365-2648.1995.21010103.x

Hibbard, J.H. and Mahoney, E. (2010), "Toward a theory of patient and consumer activation", Patient Education and Counseling, Vol. 78 No. 3, pp. 377-381. https://doi.org/10.1016/j.pec.2009.12.015

Jean, C., Stal-Le Cardinal, J., Jankovic, M., Bocquet, J.-C. and Espinoza, P. (2012), "Telehealth: Towards a Global Industrial Engineering Framework Based on Value Creation for Healthcare Systems Design", DS 70: Proceedings of DESIGN 2012 / the 12th International Design Conference, Dubrovnik, Croatia, pp. 949958.

Joseph-Williams, N., Elwyn, G. and Edwards, A. (2014), "Knowledge is not power for patients: A systematic review and thematic synthesis of patient-reported barriers and facilitators to shared decision making", Patient Education and Counseling, Vol. 94 No. 3, pp. 291-309. https://doi.org/10.1016/j.pec.2013.10.031

Katon, W.J., Lin, E.H.B., Von Korff, M., Ciechanowski, P., Ludman, E.J. et al. (2010), "Collaborative Care for Patients with Depression and Chronic Illnesses", New England Journal of Medicine, Vol. 363 No. 27, pp. 2611-2620. https://doi.org/10.1056/NEJMoa1003955

Kim, J.Y., Wineinger, N.E., Taitel, M., Radin, J.M., Akinbosoye, O. et al. (2016), "Self-monitoring utilization patterns among individuals in an incentivized program for healthy behaviors", Journal of Medical Internet Research, Vol. 18 No. 11. https://doi.org/10.2196/jmir.6371

McColl, L.D., Rideout, P.E., Parmar, T.N. and Abba-Aji, A. (2014), "Peer support intervention through mobile application: An integrative literature review and future directions.", Canadian Psychology/Psychologie canadienne, Vol. 55 No. 4, pp. 250-257. https://doi.org/10.1037/a0038095

Mead, N. and Bower, P. (2000), "Patient-centredness: a conceptual framework and review of the empirical literature", Social Science \& Medicine, Vol. 51 No. 7, pp. 1087-1110. https://doi.org/10.1016/S02779536(00)00098-8

Merriam-Webster (2017), Collaborate. [online] Merriam-Webster. Available at: https://www.merriamwebster.com/dictionary/collaboration (accessed: 05.12.2017).

Patou, F. and Maier, A.M. (2017), "Engineering Value-Effective Healthcare Solutions: A Systems Design Perspective", International Conference on Engineering Design (ICED17), pp. 31-40.

Paz Castro, R., Haug, S., Filler, A., Kowatsch, T. and Schaub, M.P. (2017), "Engagement Within a Mobile PhoneBased Smoking Cessation Intervention for Adolescents and its Association With Participant Characteristics and Outcomes", Journal of Medical Internet Research, Vol. 19 No. 11, pp. e356. https://doi.org/10.2196/jmir.7928

Pfaff, K. and Markaki, A. (2017), "Compassionate collaborative care: an integrative review of quality indicators in end-of-life care", BMC Palliative Care, Vol. 16 No. 1, pp. 65. https://doi.org/10.1186/s12904-017-02464

Pirolli, P., Mohan, S., Venkatakrishnan, A., Nelson, L., Silva, M. and Springer, A. (2017), "Implementation Intention and Reminder Effects on Behavior Change in a Mobile Health System: A Predictive Cognitive Model”, Journal of Medical Internet Research, Vol. 19 No. 11, pp. e397. https://doi.org/10.2196/jmir.8217

Pravettoni, G. and Gorini, A. (2011), “A P5 cancer medicine approach: Why personalized medicine cannot ignore psychology", Journal of Evaluation in Clinical Practice, Vol. 17 No. 4, pp. 594-596. https://doi.org/10.1111/j.1365-2753.2011.01709.x

Sclafani, J., Tirrell, T.F. and Franko, O.I. (2013), "Mobile tablet use among academic physicians and trainees", Journal of Medical Systems, Vol. 37 No. 1. https://doi.org/10.1007/s10916-012-9903-6

Stewart, M., Brown, J.B., Weston, W.W., McWhinney, I.R., McWilliam, C.L. and Freeman, T.R. (2003), "PatientCentred Medicine: transforming the clinical method", Quality in Primary Care, Vol. 11, pp. 332-333.

Thompson, A.G.H. (2007), "The meaning of patient involvement and participation in health care consultations: A taxonomy", Social Science and Medicine, Vol. 64 No. 6, pp. 1297-1310. https://doi.org/10.1016/j.socscimed.2006.11.002

Thorpe, J.R., Forchhammer, B.H. and Maier, A. (2016), "Needs elicitation for novel pervasive healthcare technology", Proceedings of International Design Conference, DESIGN, DS 84, pp. 1947-1956.

Thorpe, J.R., Forchhammer, B.H. and Maier, A. (2017), "Sensing behaviour in healthcare design", DS 87-3 Proceedings of the 21st International Conference on Engineering Design (ICED 17), Vol 3: Product, Services and Systems Design, Vancouver, Canada, August 21-25, 2017, pp. 171-180.

Twine Health (2016), The New Behavior Change Model, Chris Torrer. 
Weinhold, I. and Gastaldi, L. (2015), "From Shared Decision Making to Patient Engagement in Health Care Processes: The Role of Digital Technologies", In: Gurtner, S. and Soyez, K. (Eds.), Challenges and Opportunities in Health Care Management, Springer International Publishing, Cham, pp. 185-196. https://doi.org/10.1007/978-3-319-12178-9_15

Julie Falck Valentin-Hjorth, PhD student

Technical University of Denmark, DTU Management Engineering

Produktionstorvet, byg 424, 2800 Lyngby, Denmark

Email: julf@dtu.dk 